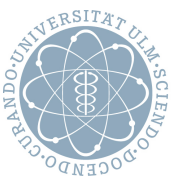

\title{
A Multicopter-Based Focusing Method for Ground Penetrating Synthetic Aperture Radars
}

Markus Schartel, Krishnendhu Prakasan, Philipp Hügler, Ralf Burr, Winfried Mayer, and Christian Waldschmidt

(C) 2018 IEEE. Personal use of this material is permitted. Permission from IEEE must be obtained for all other uses, in any current or future media, including reprinting/republishing this material for advertising or promotional purposes, creating new collective works, for resale or redistribution to servers or lists, or reuse of any copyrighted component of this work in other works.

DOI: 10.1109/IGARSS.2018.8518905 


\title{
A MULTICOPTER-BASED FOCUSING METHOD FOR GROUND PENETRATING SYNTHETIC APERTURE RADARS
}

\author{
Markus Schartel ${ }^{*}$, Krishnendhu Prakasan ${ }^{*}$, Philipp Hügler ${ }^{*}$, Ralf Burr $^{\dagger}$, Winfried Mayer ${ }^{\ddagger}$, \\ and Christian Waldschmidt*
}

\author{
*Institute of Microwave Engineering, Ulm University, 89081, Germany \\ †Ulm University of Applied Sciences, 89075 Ulm, Germany \\ ${ }^{\ddagger}$ Endress+Hauser GmbH+Co. KG, 79689 Maulburg, Germany \\ Email: markus.schartel@uni-ulm.de
}

\begin{abstract}
A subsurface focusing method for multicopter-based mine detection using a ground penetrating synthetic aperture radar (GPSAR) is presented. In the first part of this paper, the challenges of a side-looking single-input single-output (SISO) GPSAR operating in stripmap mode are highlighted by simulation results. As verification, rail-based frequencymodulated continous-wave (FMCW) GPSAR measurement are shown.

In the second part of this paper, the 3D imaging circular SAR (CSAR) approach for subsurface focusing is discussed. It is shown that the $3 \mathrm{D}$ position of buried point-like targets can be determined unambiguously. As proof of concept simulation results as well as first multicopter-based CSAR measurements are presented.
\end{abstract}

Index Terms - Ground penetrating synthetic aperture radar, CSAR, unmanned aerial vehicle, mine detection

\section{INTRODUCTION}

Anti-personnel landmines are hidden weapons, which stay a permanent threat to civilians after hostile actions [1]. According to the landmine monitor [2] the number of casualties is rising again. In 2016 more than 8600 people were killed or injured by landmines, unexploded cluster munitions, and other explosive remnants of war.

Due to the low metal content in modern mines, ground penetrating radars (GPRs) are a promising complement to metal detectors. Presently, most GPR sensors are hand-held devices which are operated very close to the surface inside the hazardous area.

In [3] a vehicle-based multiple-input multiple-output (MIMO) GPSAR with a side-looking geometry operating in stripmap mode is presented. The advantages of this system approach compared to state-of-the-art downward looking close-to-ground GPRs are the increased area throughput and the possibility to scan from a safe distance. After performing a single stripmap SAR measurement, multiple 2D radar

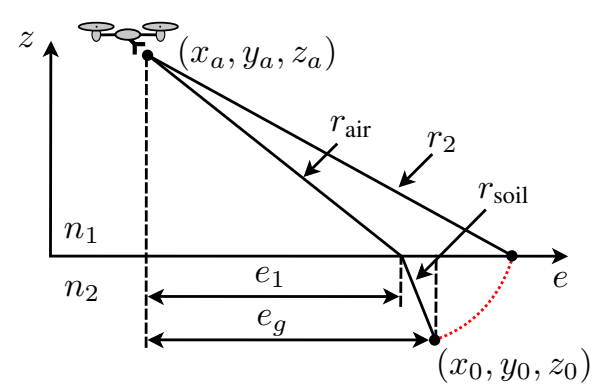

Fig. 1. Illustration of the geometry at the transition between air and soil according to Snell's law in 3D space. $e$ represents the coordinate $x$ or $y$.

images for different depths, so called C-scans or tomograms, can be processed [4].

Unfortunately, the areas to be investigated are often difficult to access by vehicles. When considering the dimensions of the vehicle-based system it is clear, that such a MIMO system cannot be mounted on a commercially available multicopter. As a compromise a bistatic, light weight, FMCW GPSAR has been developed $[5,6]$.

To determine the $3 \mathrm{D}$ position of buried objects from one stripmap SAR measurement is difficult, which is a drawback of the SISO system approach. However, this problem can be overcome by performing cross-track SAR interferometry [7]. For this purpose, the area under investigation has to be overflown several times at slightly different heights.

Another approach is to exploit the flexibility of the multicopter with respect to its trajectory. In this paper it is shown, that the $3 \mathrm{D}$ position of buried targets can be determined with CSAR [8], whereby the UAV flies a circle above the area of interest with the antenna pointing to its center.

The paper is organized as follows: In Section II the sidelooking stripmap GPSAR theory as well as rail-based GPSAR measurements are discussed. Simulation and measurement results for circular GPSAR are presented in Section III, and Section IV gives a short conclusion. 


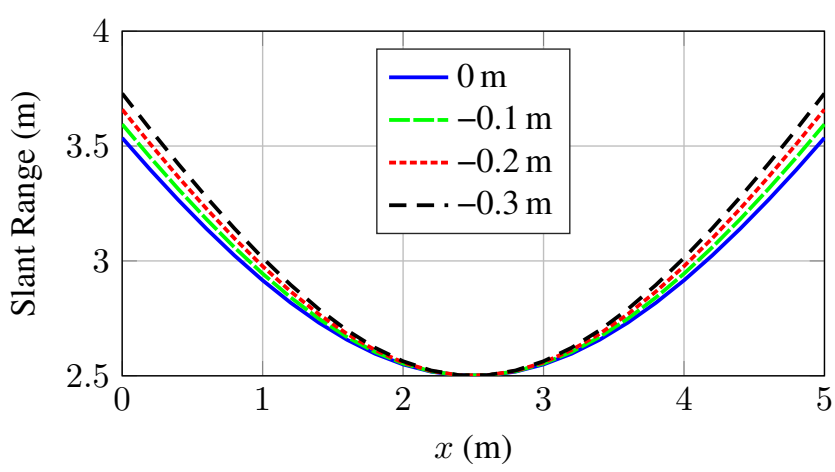

Fig. 2. Distance between the radar and targets buried in different depths with the same electrical path length at the PCA in stripmap mode.

\section{STRIPMAP GPSAR}

SAR uses the proper motion of a side-looking radar to generate high-resolution 2D images. In stripmap mode the radar is moved in a constant altitude along a straight trajectory. However, for subsurface SAR processing the depth $z$ is an additional variable, that cannot be neglected.

According to Snell's law an electromagnetic wave is refracted at the interface between air and soil as shown in Fig. 1. As explained in detail in [4], the task for subsurface processing is to calculate the intersection distance $e_{1}$ for each pixel $\left(x_{0}, y_{0}, z_{0}\right)$ of the 2D radar image, where $z_{0}$ denotes the focus plane. The ambiguity, which needs to be resolved, is illustrated by the red dotted line. All pixels placed on this line have the same path length $r_{2}=r_{\text {air }}+r_{\text {soil }}$.

In Fig. 2 the distance $r$ between the radar and 4 buried targets is shown. The radar was moved in $x$-direction in an altitude of $1.5 \mathrm{~m}$. At the point of closest approach (PCA) the path length for all targets is $r=2.5 \mathrm{~m}$. It can be recognized, that the hyperbolas of deeper buried targets have smaller opening angles. In theory a differentiation is possible, but in the case of measurement the range resolution as well as the visibility of the targets are limited.

Figure 3 shows simulation results for GPSAR with a bandwidth of $B=3 \mathrm{GHz}$ and a $3 \mathrm{~dB}$-beamwidth of $\theta_{\mathrm{az}}=60^{\circ}$. The radar was moved in an altitude of $1.5 \mathrm{~m}$ in $x$-direction (cross-range). The 4 targets have all been placed in a constant $y$-distance (ground-range) of $2.5 \mathrm{~m}$ and in different depths. The soil is modelled as homogeneous half-space.

The corresponding 2D radar image after SAR processing using a back-projection algorithm is depicted in Fig. 4. This image represents a slice of a 3D tomogram at the focus plane $z=0$. It should be noted, that targets in an other depth than the focus plane are shifted in $y$-direction and blurred, but they do not vanish. In a real environment with extended targets and clutter it will be difficult to detect this deformation in the target response. Thus, the image has to be processed for

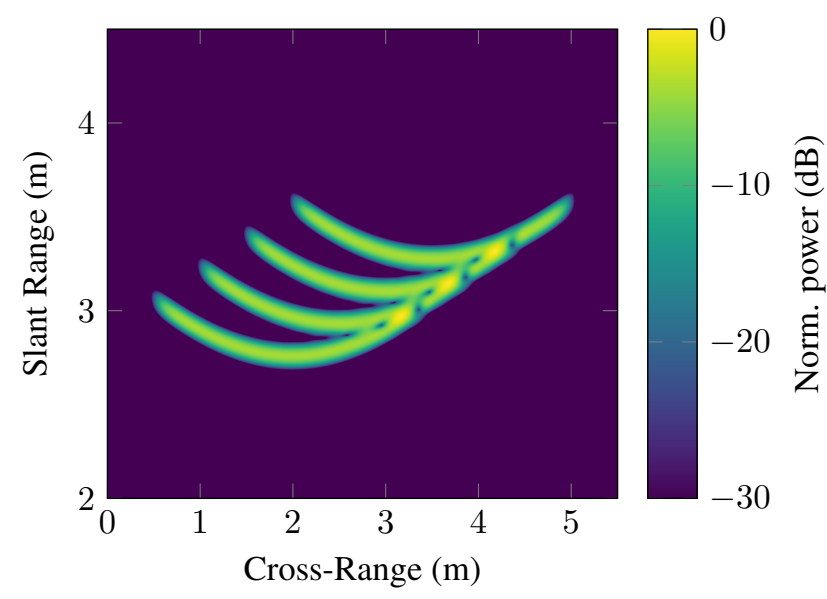

Fig. 3. Simulated range compressed radar data for 4 targets in the same $y$-distance and in a depth of $z=0$ (left) to $z=-0.3 \mathrm{~m}$ (right) in steps of $\Delta z=0.1 \mathrm{~m}\left(\varepsilon_{r}=5\right)$.

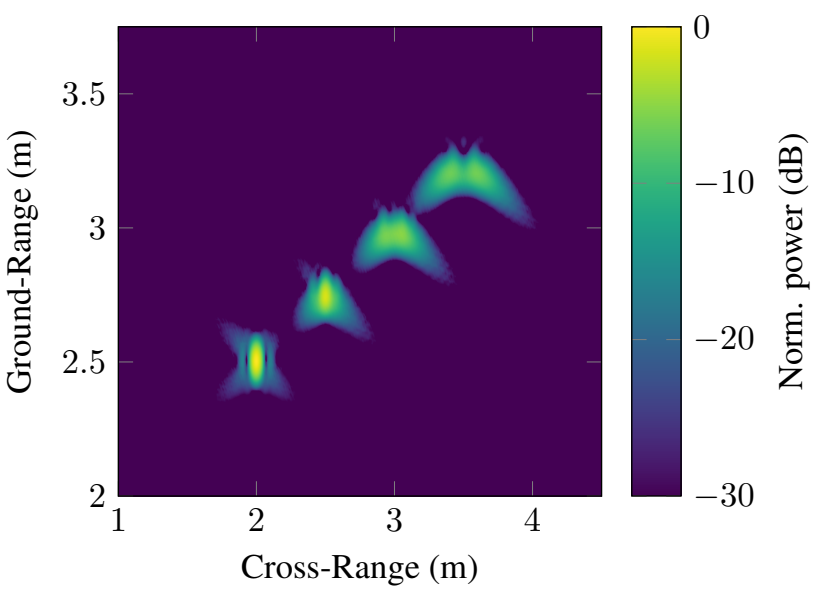

Fig. 4. Back-projection of the the data shown in Fig. 3. The focus plane is at $z=0\left(\varepsilon_{r}=5\right)$.

different depths, and the maximum SNR of each target has to be detected to determine its depth.

As verification rail-based stripmap GPSAR measurements were performed. In Fig. 5 the measurement setup is shown. The bistatic FMCW GPSAR was equipped with logarithmic periodic dipole antennas (LPDAs). The radar was moved in an altitude of approximately $1.5 \mathrm{~m}$ parallel to the test field.

As targets 4 corner reflectors have been buried in a depth of $z=0$ (left) to $z=-0.3 \mathrm{~m}$ (right) in steps of $\Delta z=0.1 \mathrm{~m}$. The 4 reflectors on the edges are used to mark the boarders of the test field. The RCS value of the reflectors is approximately $\sigma=-12.2 \mathrm{dBsm}$. The permittivity of the sand $\left(\varepsilon_{r} \approx 3\right)$ was estimated using the knowledge of the actual target depth.

In Fig. 6 the 2D radar image for the depth $z=0 \mathrm{~m}$ is shown. The 4 reflectors marking the edge of the measure- 


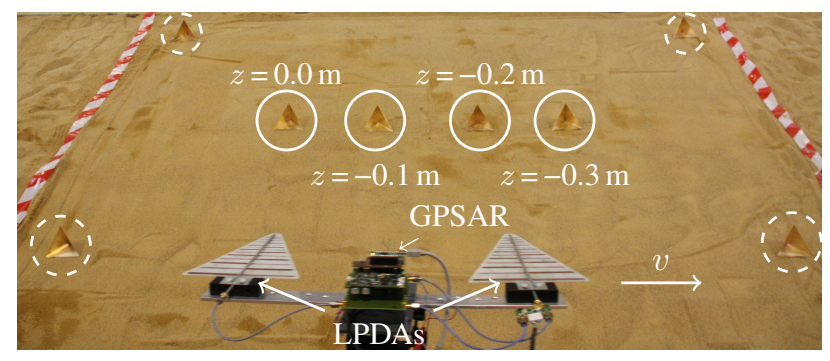

Fig. 5. Photo of the GPSAR sensor module and the measurement setup.

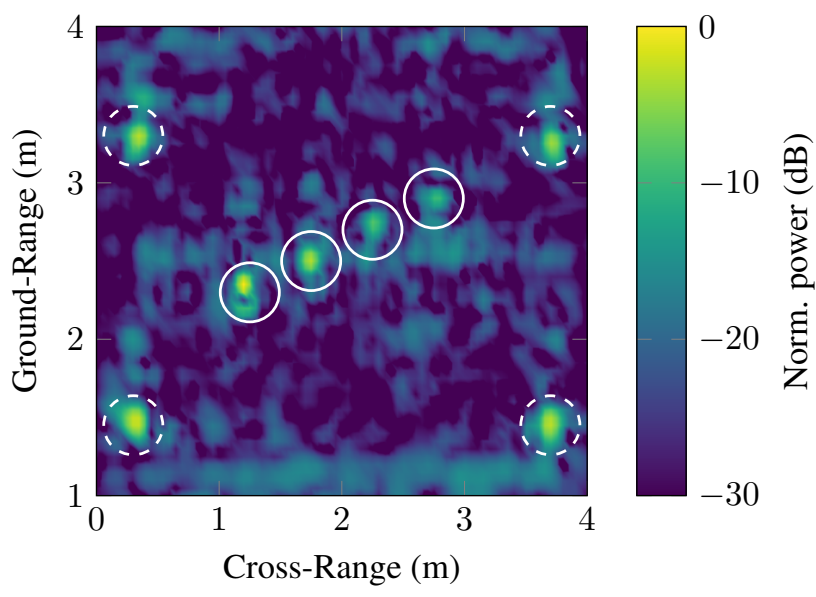

Fig. 6. Back-projection for the focus plane $z=0 \mathrm{~m}$ of the setup shown in Fig. $5\left(\varepsilon_{r}=3\right)$.

ment field as well as the 4 reflectors in the center of the image can be detected. Due to the material loss, the signalto-clutter ratio (SCR) is decreasing with increasing depth. As expected, the targets in a different depth are simply shifted in $y$-direction. A deformation of the target response can not be observed. The position in $x$-direction can be determined, but the ambiguity in $y$-distance and the target depth $z$ cannot be resolved without investigating the small changes in the signal-to-noise ratio (SNR) of the targets for different layers.

\section{CIRCULAR GPSAR}

To improve the position determination the idea is to use the 3D imaging capabilities of CSAR for subsurface processing. Instead of flying a straight line, the UAV can fly circles above the area of interest. Thus, the target response would be spread over a circle, if the focus lies on a different depth than the focus plane. A crucial requirement for CSAR is the angular persistence of the target for 3D localization. Regarding the dimensions and the shape of anti-personnel mines this requirement could be fulfilled.

In Fig. 7 a simulation result for circular GPSAR is shown. The altitude of the radar is $z_{a}=1.5 \mathrm{~m}$ and the diameter of the

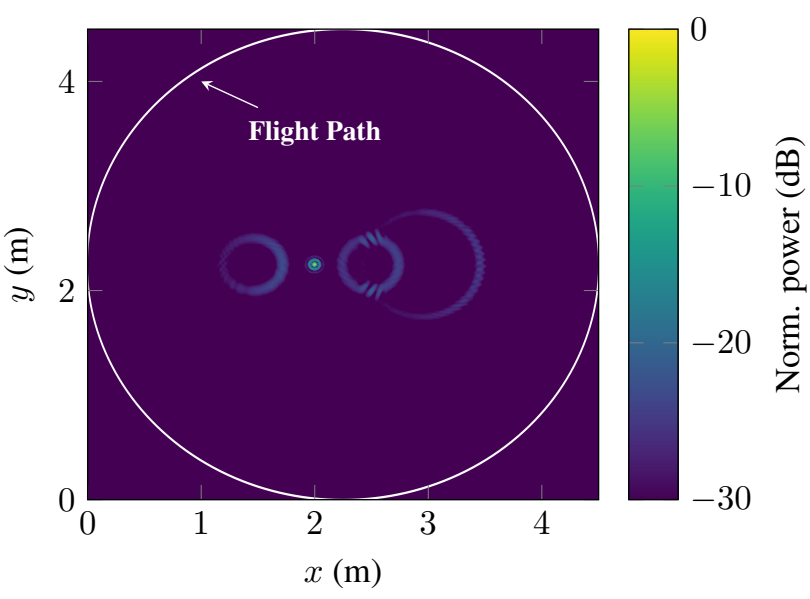

Fig. 7. Back-projection for the focus plane $z=-0.1 \mathrm{~m}$ $\left(\varepsilon_{r}=5\right)$.

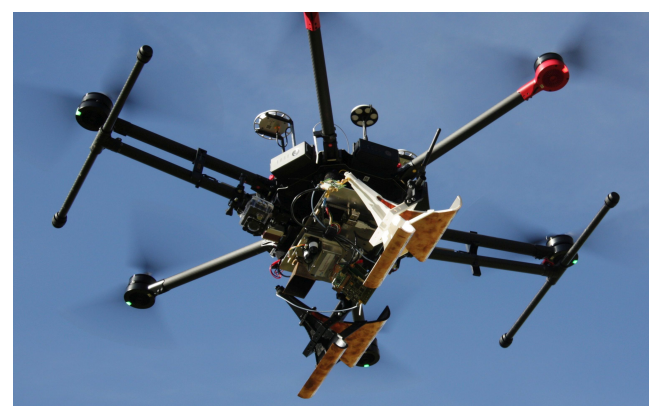

Fig. 8. Measurement system consisting of a multicopter, a GPSAR, a radar and lidar altimeter, a real time kinematic global navigation satellite system (RTK GNSS), and a data logger.

circle flown is $4.5 \mathrm{~m}$. The targets are visible over $360^{\circ}$ and they are placed in a depth of $z=0$ (left) to $z=-0.3 \mathrm{~m}$ (right) in steps of $\Delta z=0.1 \mathrm{~m}$. As expected, the target responses from targets not in the focus plane are spread over a circle. The diameter of these circles depends on the difference between the focus plane and the depth of the target.

For the proof of concept circular GPSAR measurements were performed with the system shown in Fig. 8. As targets two corner reflector have been placed in different heights above the surface pointing upwards. Additionally, an earth rod was placed in the test field as shown in Fig. 9. The multicopter was autonomously flying a circle with a diameter of $10 \mathrm{~m}$ in an altitude of about $5 \mathrm{~m}$.

The first measurement results from circular overflights are shown in Fig. $10(z=0 \mathrm{~m})$ and Fig. $11(z=1 \mathrm{~m})$. Both images are normalized to their maximum. Despite the limited image quality caused by pointing errors of the antenna, this result is a proof of concept for the circular GPSAR-approach. A 3D position determination for point like targets is possible. The target response is spread over a circle, if the focus plane and 


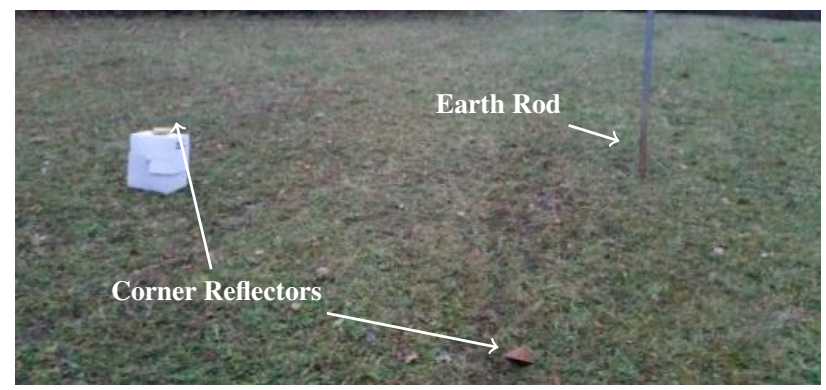

Fig. 9. Measurement setup for CSAR measurements.

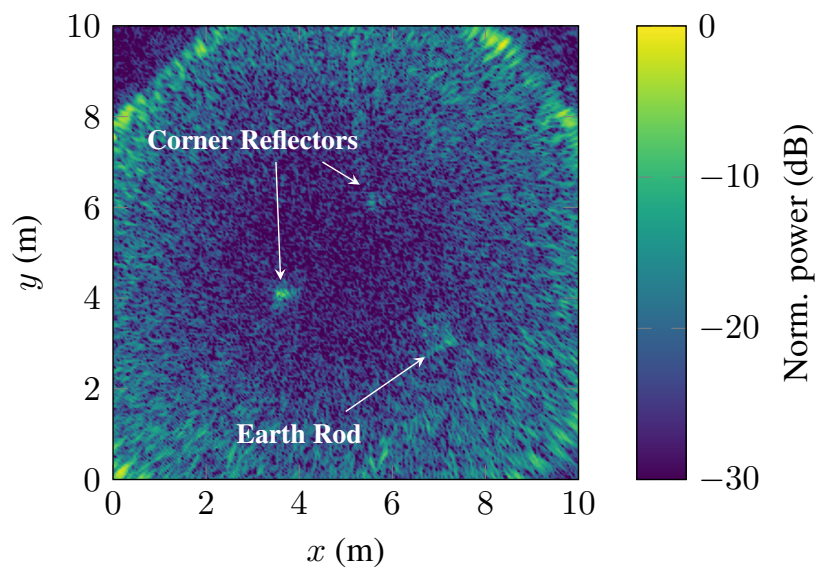

Fig. 10. Back-projection for the focus plane $z=0 \mathrm{~m}$.

the target depth differ.

\section{ACKNOWLEDGEMENT}

The authors would like to thank the Urs Endress foundation for their support and the collaboration.

\section{CONCLUSION}

A subsurface focusing method for GPSAR using the trajectory of a UAV has been presented. The problems of stripmap SAR imaging have been highlighted with simulation and measurement results. As a solution a circular GPSAR approach has been introduced, in which the responses of targets outside the focus plane are spread over circles. The approach has been verified by simulations and experimental tests.

\section{REFERENCES}

[1] D. J. Daniels and Institution of Electrical Engineers, Ground Penetrating Radar, Electromagnetics and Radar Series. Institution of Engineering and Technology, 2004.

[2] International Campaign to Ban Landmines, Landmine Monitor 2017, Human Rights Watch.

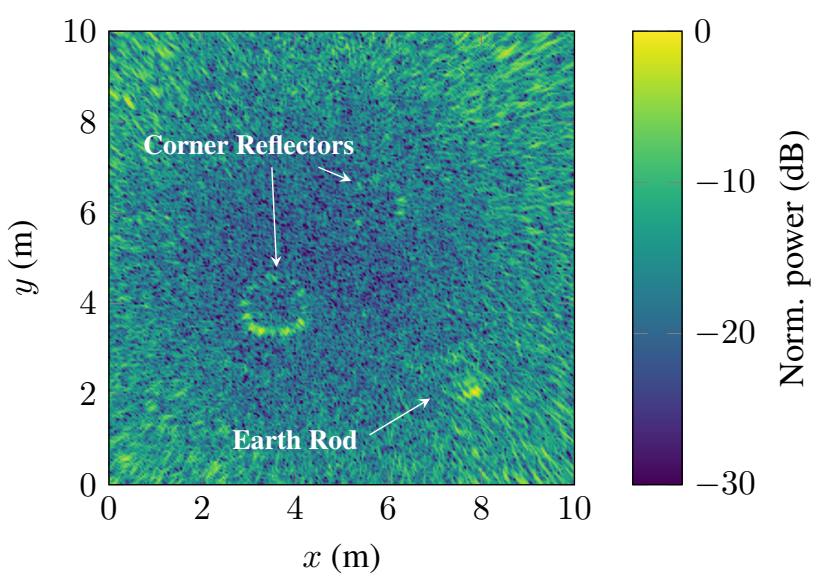

Fig. 11. Back-projection for the focus plane $z=1 \mathrm{~m}$.

[3] M. Peichl, E. Schreiber, A. Heinzel, and T. Kempf, "TIRAMI-SAR - a synthetic aperture radar approach for efficient detection of landmines and UXO," in EUSAR; 10th European Conference on Synthetic Aperture Radar, June 2014, pp. 1-4.

[4] A. Heinzel, M. Peichl, E. Schreiber, F. Bischeltsrieder, S. Dill, S. Anger, T. Kempf, and M. Jirousek, "Focusing Methods for Ground Penetrating MIMO SAR Imaging within Half-Spaces of Different Permittivity," in Proceedings of EUSAR: 11th European Conference on Synthetic Aperture Radar, June 2016, pp. 1-5.

[5] M. Schartel, R. Burr, W. Mayer, N. Docci, and C. Waldschmidt, "UAV-Based Ground Penetrating Synthetic Aperture Radar," in IEEE MTT-S International Conference on Microwaves for Intelligent Mobility (ICMIM), 2018.

[6] R. Burr, M. Schartel, P. Schmidt, W. Mayer, T. Walter, and C. Waldschmidt, "Design and Implementation of a FMCW GPR for UAV-based Mine Detection," in IEEE MTT-S International Conference on Microwaves for Intelligent Mobility (ICMIM), 2018.

[7] G. Krieger, I. Hajnsek, K. P. Papathanassiou, M. Younis, and A. Moreira, "Interferometric Synthetic Aperture Radar (SAR) Missions Employing Formation Flying," Proceedings of the IEEE, vol. 98, no. 5, pp. 816843, May 2010.

[8] O. Ponce, P. Prats-Iraola, M. Pinheiro, M. RodriguezCassola, R. Scheiber, A. Reigber, and A. Moreira, "Fully Polarimetric High-Resolution 3-D Imaging With Circular SAR at L-Band," IEEE Transactions on Geoscience and Remote Sensing, vol. 52, no. 6, pp. 3074-3090, June 2014. 